\title{
Resiliencia ambiental y social a través del diseño: Universidad Verde y Sustentable (USEN)
} Environmental and Social Resilience through Design: Green and Sustainable University (USEN)

\author{
Mario Yadir Rendón Sallard \\ Universidad de Sonora, México \\ yadir42@hotmail.com
}

\author{
Patricia Sandoval Murillo \\ Universidad de Sonora, México \\ psandoval79@yahoo.com.mx
}

\begin{abstract}
Universidad Verde y Sustentable [Green and Sustainable University] (USEN) is a multidisciplinary project carried out at the Universidad de Sonora that emerged from a national program where international specialists trained university professors in applied leadership, energy efficiency and renewable energies. The project integrates architecture, design, landscaping, energy efficiency, technology and sustainability and includes the technical training of professionals, university students, teachers and the community, as well as the design and promotion of a social marketing campaign that seeks to create a paradigm change in the consumption of energy resources, environmental care, the use of renewable energies, sustainability and resilience.
\end{abstract}

Keywords: Cambio climático; Eficiencia energética; Energías renovables; Diseño resiliente; Diseño social.

\section{Introducción}

El proyecto Universidad Verde y Sustentable (USEN) fue diseñado para implementarse como programa piloto dentro del Departamento de Arquitectura y Diseño de la Universidad de Sonora (ubicada en Hermosillo, Sonora, México). Es un proyecto multidisciplinario que fue diseñado para implementar estrategias que fomenten hábitos de ahorro y eficiencia en los recursos y la utilización de energías renovables, incluyendo una campaña que fomenta una mayor conciencia ambiental y sensibilidad hacia la responsabilidad social dentro de la comunidad universitaria. Este artículo describe la motivación inicial del proyecto, las actividades que se han ido realizando en respuesta a esa motivación, la manera en que las actividades han ido evolucionando en respuesta a las necesidades detectadas, y la necesidad de evaluar la campaña, así como la metodología propuesta para realizar esta evaluación.

\section{Antecedentes}

Este proyecto inició con una propuesta realizada para el diplomado "Liderazgo Aplicado en Eficiencia Energética y Energías Renovables" impartido por la Escuela de Salud Pública de la Universidad de Harvard, InTrust Global Investments y la Secretaría de Energía de México durante 2014-2015 en la Unidad Regional Centro de la Universidad de Sonora. El objetivo de este diplomado fue generar proyectos en los que se impulsara la eficiencia energética en espacios interiores y exteriores, así como la utilización de energías renovables, para contrarrestar los efectos del calentamiento global. Este artículo describe las dos etapas que se han desarrollada a partir de la propuesta inicial (Rendón, 2015), así como las prioridades de seguimiento que se han establecido con base en los resultados de las actividades realizadas a la fecha.

\section{Justificación}

En reconocimiento de la necesidad urgente de implementar acciones para contrarrestar el cambio climático desde diferentes ámbitos y disciplinas (ONU, 2015; INECC, s.f.), este proyecto parte del compromiso de formar diseñadores que puedan reconocer las necesidades sociales y ambientales que vivimos y actuar a favor de generar un beneficio para la comunidad (Frascara, 2004). En su libro Green Graphic Design, Brian Dougherty identifica tres maneras distintas de pensar en el papel de un diseñador: el diseñador como manipulador de cosas; el diseñador como fabricante de mensajes; y el diseñador como agente de cambio (Dougherty, 2008, p. 8). El proyecto Universidad Verde y Sustentable busca formar tanto a diseñadores gráficos como a arquitectos (y estudiantes de otras disciplinas) que se asuman como agentes de cambio, integrándolos a un proyecto que les dará una experiencia de relevante importancia en su formación como futuros profesionistas, ya que se están enfrentando a un proyecto real que propone soluciones a un problema urgente. Los estudiantes tendrán la oportunidad de probar sus habilidades y conocimientos, logrando ver en su comunidad los resultados de su trabajo.

\section{Marco Conceptual}

Los beneficios sociales de este proyecto se definen claramente en su programa por tratarse de un tema de diseño participativo cuyo último objetivo es promover la resiliencia de la comunidad universitaria.

Tal y como describe Calvente (2007, p.2), "el concepto de resiliencia tiene tres características que lo definen: la cantidad de cambio o transformaciones que un sistema complejo puede soportar manteniendo las mismas propiedades funcionales y estructurales; el grado en el que el sistema es capaz de autoorganizarse; y la habilidad del sistema complejo para 
desarrollar e incrementar la capacidad de aprender, innovar y adaptarse“.

La resiliencia es a la vez una teoría de cómo los sistemas pueden comportarse, una práctica o abordaje proactivo para la planificación, y una herramienta analítica que permite examinar cómo y por qué algunos sistemas son más capaces de responder a los trastornos (Vale, 2014). El desarrollo de la resiliencia requiere factores de protección tanto externos como internos, que incluyen: cooperación y comunicación, empatía, habilidades para la resolución de problemas, metas y aspiraciones bien definidas, alta autoeficacia y la autoconciencia que se desarrollan naturalmente en respuesta a factores de protección ambiental, y contribuyen a un desarrollo académico, social y de salud (Jowkar et al., 2014, p. 33).

La resiliencia y la sustentabilidad están directamente relacionadas con las influencias en el largo plazo de las consecuencias de las transformaciones y el cambio de todo sistema complejo (Calvente, A. 2007). Cada comunidad tiene sus propias características, necesidades, retos y soluciones potenciales, lo cual requiere el desarrollo de su propia visión, programas y planes de acción para construir resiliencia, con el fin de promover el auto-conocimiento, acciones que generen resultados medibles, y un plan implementable y sustentable (Edwards, 2011).

Existen sinergias y eficiencias que se pueden obtener si se aprovecha el proceso de planificación de un campus universitario para abordar temas de resiliencia y mitigar los daños del cambio climático. Esto requiere no sólo incluir en el presupuesto universitario un rubro para atender los riesgos del cambio climático, sino establecer un proceso reiterativo que incluya recopilar datos y convocar a las partes interesadas para establecer necesidades y desarrollar metas, explorar direcciones posibles para la inversión y el desarrollo del campus, y refinar la propuesta para generar próximos pasos (Foster y Smith, 2015).

El concepto de diseño participativo establece la relación de la comunidad afectada o implicada dentro del proyecto - la cual se ha convertido en un reto en los últimos años en acciones similares- pues la participación activa de las personas involucradas resulta fundamental en este tipo de programas. Se ha comprobado que cuando la comunidad afectada no se involucra y se llevan a cabo acciones o proyectos sin tomar en cuenta las necesidades de la comunidad (tales como la concientización o incluso la capacitación en el tema) es altamente probable que el proyecto no funcione pues la gente no se sentirá incluida. (Bas y Guillo, 2015).

Un reto de este proyecto es promover mecanismos efectivos de participación, sobre todo de los actores más vulnerables, entendiendo que la participación no se limita a informar o consultar; si bien es necesario estar informado para participar, la participación es una interacción y conlleva formar alianzas, hacer planificación conjunta, delegar el poder y fomentar el empoderamiento y el control ciudadano, en este caso de los distintos actores o partes interesadas al interior de la universidad (Fernández, 2014). La participación como sujeto de investigación e incluso en la implementación de la tecnología que se propone como parte de este proyecto no sería suficiente, pues cuando se trata de evaluar los procesos de toma de decisiones, puede generarse un distanciamiento entre las propuestas y las necesidades de la institución, sus objetivos en cuanto al proyecto y los usuarios, en este caso la comunidad universitaria. Ese distanciamiento sería un factor de fracaso para un proyecto de esta naturaleza (Bas y Guillo, 2015).

\section{Actividades}

A partir de la propuesta inicial (Rendón, 2015), el proyecto ha ido evolucionando según las barreras y oportunidades que se han encontrado y tomando en cuenta los aprendizajes que han permitido establecer prioridades de acción.

\section{Análisis de consumo energético y propuesta de cambios a la infraestructura}

Inicialmente el proyecto contempló la implementación de tecnología para la generación de energía renovable -como paneles solares - y sistemas electrónicos de ahorro energético basados en eficientar la manera en que se consume la energía eléctrica dentro del campus universitario.

Se realizó un análisis del consumo energético del campus y un estudio de la idiosincrasia de los usuarios (entrevistas con autoridades, alumnos, docentes y personal universitario), y se hicieron recomendaciones para mejorar la infraestructura y generar un aumento en la eficiencia energética, incluyendo cambio de lámparas LED, instalación de aires acondicionados de alta eficiencia, automatización de sistemas y creación de más áreas verdes. Durante el análisis de la infraestructura actual con que cuenta la Universidad de Sonora, se observó que la gran mayoría de sus edificios cuentan con problemas de diseño arquitectónico que influyen directamente en un uso inadecuado y desmedido de energía eléctrica y por consecuencia de altos costos económicos. Aunado a esto, existe una nula cultura en el cuidado del uso eficiente de la energía por parte de sus usuarios, lo cual también provoca aumentos en los costos e impactos en la salud y aprovechamiento laboral y académico.

Se contó con la participación de una empresa especializada en paneles solares que realizó un análisis del consumo energético del Departamento de Arquitectura y Diseño y se propuso la instalación de 400 paneles fotovoltaicos que cubrirían la totalidad de la demanda energética requerida $(100 \mathrm{KW})$ del edificio -como se observa en la Figura 1-, generando un excedente de energía que potencialmente se podría vender a empresas o instituciones cercanas al campus universitario, aumentando el impacto social y ambiental puesto que la energía proviene de fuentes renovables. 


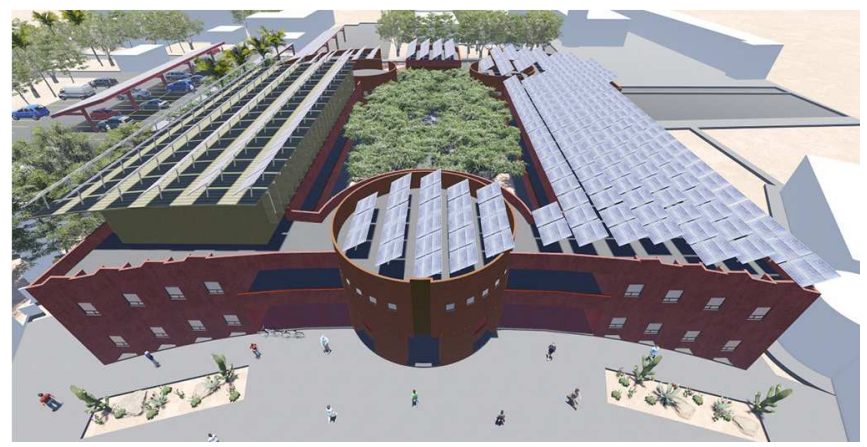

Figura 1: Vista aérea del edificio donde se observa el arreglo de paneles fotovoltaicos en azoteas.

En entrevistas con autoridades universitarias se determinó que la inversión necesaria para la instalación de los paneles fotovoltaicos era demasiado elevada, y que además existe una resistencia a la implementación de esta tecnología por parte del personal que le tendría que dar mantenimiento a los paneles. Fue necesario buscar otras alternativas, por ejemplo: el cambio de focos incandescentes por focos LED; ya que al usar focos incandescentes de 100 Watts se consume 1 kilowatt-hora por cada 10 horas de uso. La iluminación puede representar una tercera parte del consumo de energía eléctrica en un espacio como el aula universitaria. En el caso de los sistemas de climatización, el gasto aumenta radicalmente ya que es uno de los sistemas de mayor consumo eléctrico, y estos equipos resultan indispensables en climas cálidos como el de la localidad donde se ubica el proyecto. Por lo tanto, la alternativa de cambio de focos incandescentes por LED e instalación de sistemas de climatización de alta eficiencia resultan más viables y costeables antes que la instalación de sistemas fotovoltaicos.

El uso adecuado de los sistemas de climatización existentes también puede aumentar su eficiencia, a través de pequeñas acciones como mantenerlos en una temperatura estable (es decir, no muy fría), lo cual hace que el compresor del equipo no trabaje forzadamente, cerrar las puertas de las aulas cuando se usa la climatización, y automatizar el encendido de los equipos de refrigeración. Estas acciones de uso podrían generar ahorros hasta de $20 \%$ en el consumo eléctrico (Sánchez, 2015).

Durante esta fase inicial del proyecto, los estudiantes de Arquitectura usaron sus habilidades para realizar la visualización del diseño de la infraestructura de energías renovables por medio de renders (visualizaciones fotorrealistas) que mostraron como podrían instalarse los paneles solares, así como estrategias para incrementar el área sombreada dentro del campus (Figura 2), rediseñar las áreas verdes existentes e integrar muros y azoteas verdes donde la infraestructura lo permita.

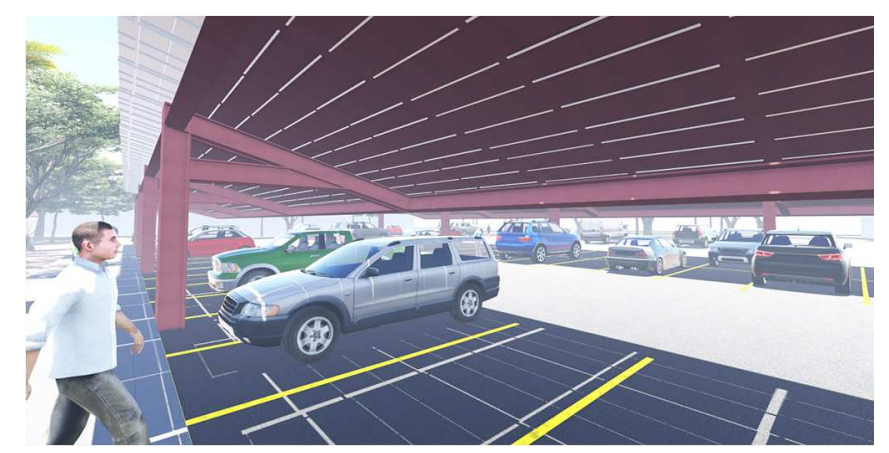

Figura 2: Además de generar energía, los paneles fotovoltaicos en estacionamientos provocan áreas de sombras.

\section{Campaña de sensibilización y concientización ambiental}

Las barreras identificadas durante el análisis de consumo energético y la elaboración de propuestas para cambios de infraestructura ilustraron claramente el "efecto rebote" que ha sido documentado por otros proyectos, donde se han implementado estrategias individuales de eficiencia energética que no resultan en una conservación de recursos (Frye-Levine, 2012). El aprendizaje de estos proyectos es que la sustentabilidad real se logra cuando existe una metodología para la negociación de la acción colectiva, cuyo punto de partida es la sensibilización acerca de la importancia de los esfuerzos de conservación.

Con este fin se formuló la campaña de concientización y educación USEN, que busca involucrar a todos los miembros de la comunidad universitaria en el esfuerzo para hacer cambios de hábitos y aumentar la responsabilidad social con su comunidad y así promover su resiliencia. Las acciones de educación ambiental que transfieren conocimientos contextualizados con el propósito de desarrollar capacidades en los actores comunitarios contribuyen a la concientización de la población y motiva a los participantes a realizar transformaciones en sus comunidades (Gallardo, 2014).

El objetivo de la campaña USEN, en su primera iteración, fue concientizar para intentar revertir y detener las consecuencias del despilfarro de energía eléctrica en la universidad. Como parte de la estrategia de comunicación se planteó la posibilidad de lograr esta integración de la comunidad universitaria, particularmente el alumnado, de dos formas: como receptor y como ejecutor. Los receptores son la parte de la comunidad a quien le llega el mensaje, y los ejecutores son la parte de la comunidad que realiza y participa activamente en la ejecución y elaboración del proyecto. Al final todos terminan participando activamente, pues como participante ejecuto, comprendo, lo vivo, y lo vuelvo a reproducir.

Una ejemplo que hemos utilizado como referencia para nuestra campaña son los materiales gráficos que el World Wildlife Fund (WWF) publica periódicamente para persuadir a la sociedad de tomar acción para controlar la contaminación y sus consecuencias, con el hashtag \#niungradomás (WWF, 2015). En esta campaña se involucra a la ciudadanía para que tomen acciones como realizar peticiones en línea para 
gestionar a los gobiernos y a las instituciones que puedan tener trascendencia en la promulgación de leyes ambientales.

Otro punto importante en esta campaña es que sea una campaña que no genere más problemas de basura y no contamine con aplicaciones impresas - como carteles y volantes - la imagen urbana del campus. Se pretende que la campaña visual sea $100 \%$ virtual $y / 0$ idear formas de promoción que no involucren impresos en papel u otro tipo de contaminantes. Actualmente se lleva a cabo por medio de la publicación de carteles en Facebook, Instagram y Twitter, y se está trabajando en la Página Web, que se pretende promover dentro del sitio oficial de la Universidad de Sonora y en las páginas de todas sus Divisiones y Departamentos.

También se han diseñado aplicaciones tipo suvenires que ayudarán a que la comunidad se relacione e identifique más con el proyecto, fungiendo así como carteles vivientes (en el caso de camisetas, carpetas y folders). En estas aplicaciones tipo suvenires se maneja la imagen del proyecto pero con elementos icónicos de la universidad (por ejemplo, los vitrales del edificio de Rectoría, Figura 3), buscando ser una manera gráfica de ligar el proyecto con la institución para darle un sentido de pertenencia. Lo que se quiere lograr con esta campaña es que la comunidad universitaria se apropie del proyecto. Deseamos influir positivamente tanto en las personas que participan en su realización como en las que reciben la información.

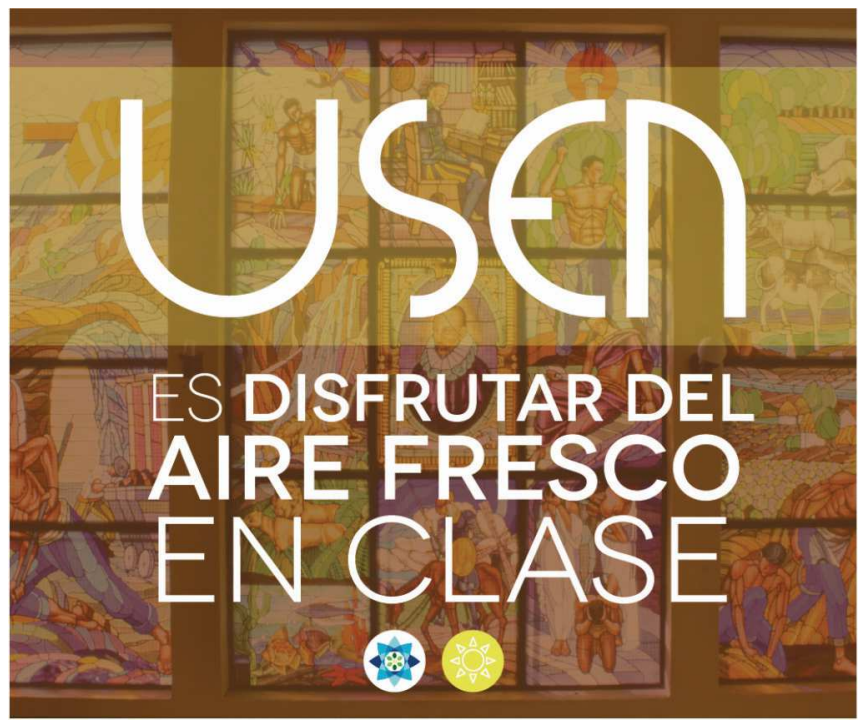

Figura 4: El uso de imágenes icónicas de la Universidad de Sonora para promover la identidad y pertenencia con el proyecto.

La campaña muestra un mensaje de las ventajas y beneficios de tener un ambiente universitario cómodo, limpio y tranquilo, y cómo hacer para logar dicho objetivo (Figura 4). La campaña está diseñada para que se presenten las publicaciones primero con la idea de identificar el beneficio, idearlo o bien idealizarlo; para luego así despertar el interés de cómo lograrlo y mantenerlo. El objetivo es interesar a la comunidad en lo que puede hacer para lograr esto como miembro de esta comunidad.

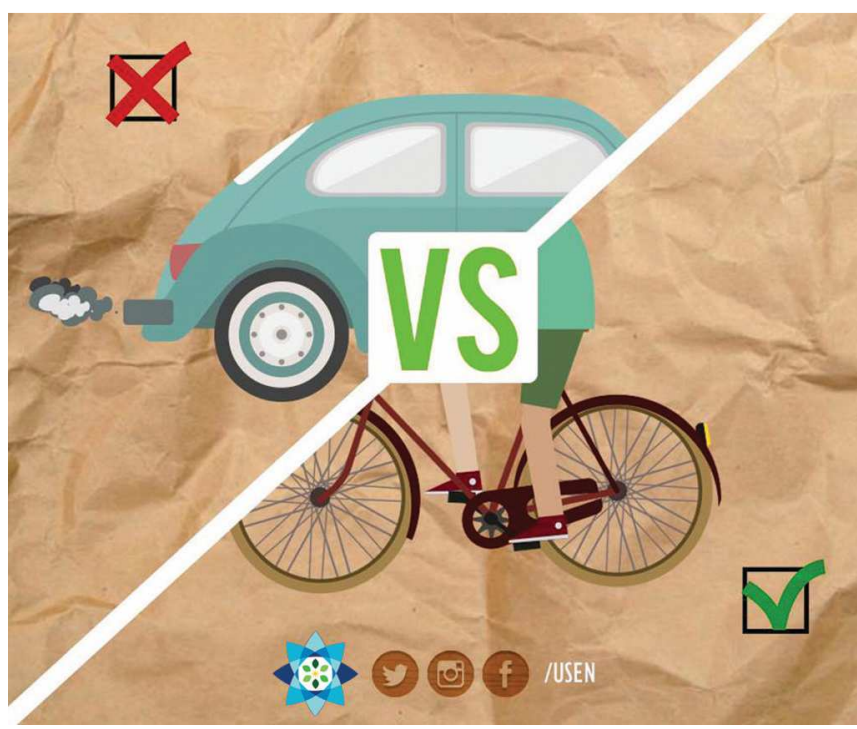

Figura 4: Ejemplo de cartel virtual para promoción en redes sociales desarrollado por los alumnos participantes.

\section{Evaluación de la campaña}

Una vez elaborada la identidad gráfica de USEN y establecidas las bases de la campaña de sensibilización, surgió la necesidad de evaluar su impacto para adecuar los mensajes transmitidos a las prioridades de la comunidad universitaria. Esta evaluación representa la próxima etapa del proyecto. Su objetivo es medir la influencia e impacto de la campaña de concientización de USEN, primero en el Departamento de Arquitectura y Diseño de la Universidad de Sonora.

Para realizar un estudio factible que arroje datos claros en cuanto a la efectividad y la influencia de la campaña en la percepción de la comunidad del Departamento de Arquitectura y Diseño, se propone utilizar métodos mixtos para realizar un proceso de evaluación rápida (Jacobson, 1997; Beebe, 2005), incluyendo la aplicación de encuestas, la realización de grupos focales y entrevistas a profundidad, entre otros. Las actividades de la evaluación rápida se realizarán en el corto, mediano y largo plazo, y se irán modificando tanto las herramientas de evaluación como las características de la campaña a partir de los datos que se vayan obteniendo.

En el corto plazo se hará una encuesta de línea base para conocer el universo con el que se está trabajando, donde se involucrará a todo aquel individuo que interactúa regularmente en este departamento: estudiantes de arquitectura y diseño gráfico, profesores de ambas licenciaturas, administrativos e intendentes, con el fin de saber cuáles son sus hábitos regulares con respecto al ahorro de recursos y determinar si la campaña inicial está teniendo un alcance favorable.

Con base en los resultados que arroje esta encuesta, se rediseñará la campaña para ser consecuentes con los cambios que los resultados sugieren y se generarán preguntas para realizar grupos focales homogéneos con actores concretos como: estudiantes de arquitectura, diseñadores, intendentes, personal administrativo, y docentes. Con estos resultados y la comparación de la medición del 
gasto energético actual versus el gasto energético de los años anteriores, se podrá observar si ha habido un cambio y cuáles pueden ser las variantes, para ir estableciendo un aproximado de la tendencia de impacto que la campaña va teniendo. Esta dinámica de evaluación rápida se podrá extender para establecer mecanismos permanentes de evaluación para poder tener resultados constantes y una medición fiable.

\section{Conclusiones}

Como lo han demostrado otras iniciativas similares a USEN, el uso de diversos medios de comunicación permite enviar un mensaje claro, conciso y efectivo sobre la manera adecuada de usar los recursos naturales y disminuir con ello los efectos medio-ambientales y de salud que provoca el consumo de combustibles fósiles. Asimismo, la implementación de sistemas y tecnologías en edificios que permitan la generación de energías limpias, el monitoreo más eficiente del consumo energético y el incremento de las áreas verdes y sombreadas en exteriores, generan un aumento en el nivel de confort térmico y en la calidad de la salud y aprovechamiento académico y laboral de los usuarios.

Se pretende que USEN se convierta en un programa modelo de eficiencia energética en el Departamento de Arquitectura y Diseño de la Universidad de Sonora por medio del cual será posible medir resultados de ahorro energético e impacto ambiental en la comunidad de usuarios, para después extenderlo a otros departamentos. La idea es que poco a poco toda persona que desee unirse al proyecto lo haga, sabiendo que su participación va a tener un fruto importante. En un mediano o largo plazo, también se espera que el proyecto se replique en otras universidades para generar una red universitaria enfocada a colaborar en proyectos similares con otras universidades del país y del mundo.

Resulta importante y urgente la participación de toda la comunidad universitaria para la solución de los problemas ambientales y de la actuación directa de especialistas que promuevan un cambio en los paradigmas culturales de esta comunidad para adoptar nuevos modelos en el uso eficiente de la energía e infraestructura universitaria. Al mismo tiempo, la integración de los estudiantes en este proyecto les dará una experiencia de relevante importancia en su formación como futuros profesionistas, del trabajo escolar al diseño, implementación y evaluación de intervenciones que van más allá de una calificación académica.

Tal y como abogan Marcus y Colding (2014), necesitamos explorar los conceptos y suposiciones fundamentales que actualmente enmarcan el discurso de la sustentabilidad y proporcionar una base más científicamente informada para los sistemas institucionales y espaciales que apoyan a los sistemas socio-ecológicos urbanos. El valor de la resiliencia para entender a las comunidades depende de tratar a las comunidades como sistemas socio-ecológicos que no son estables y deben evolucionar. Cuando los investigadores ponen a prueba la viabilidad del concepto de resiliencia para explicar situaciones reales de disputas de poder, esto revela que la resiliencia es performativa, promulgada por personas e instituciones con intereses variados y poder variable (Vale, 2014).
Se pretende que la campaña educativa de USEN vaya introduciendo a la comunidad universitaria a otros intereses como la realización de talleres, la apropiación de los espacios universitarios (incluyendo los espacios de planificación y toma de decisiones), y la promoción de cambios en el enfoque verde y social de la comunidad. Se busca sentar las bases para realizar un programa institucional de eficiencia energética y sustentabilidad. Además se busca implementar estrategias que permitan generar energía limpia y disminuir las emisiones de gases de efecto invernadero local y regionalmente. Intenta generar cambios en la conducta social y ambiental de la comunidad universitaria y del contexto que le rodea. Y finalmente, busca convertirse en un "laboratorio viviente" donde todos los usuarios participen en el cuidado, difusión y operación del proyecto.

\section{Agradecimientos}

Damos las gracias a la Secretaría de Energía de México, a InTrust Global Investments, al Centro de Salud y Ambiente Global de la Escuela de Salud Pública de Harvard y a la Universidad de Sonora por la realización del Diplomado en Liderazgo Aplicado en Eficiencia Energética y Energías Renovables. Agradecemos al Departamento de Arquitectura y Diseño y la División de Humanidades y Bellas Artes de la Universidad de Sonora por el apoyo en recursos físicos y económicos, así como a todos los alumnos y asesores que participaron en el desarrollo de este proyecto.

\section{Referencias}

Bas E. y Guillo M. (2015). Participatory foresight for social innovation. FLUX-3D method (Forward Looking User Experience), a tool for evaluating innovations. Technological Forecasting \& Social Change. 101(complete), 275-290.

Beebe, J. (2005). Rapid assessment process. En K. Kempf-Leonard (Ed.) Encyclopedia of Social Measurement. San Diego: Academic Press/Elsevier, 285-291.

Calvente, A. (2007). Resiliencia: Un concepto clave para la sustentabilidad. Complejidad y sustentabilidad. UAIS, Universidad Abierta Interamericana / Centro de Altos Estudios Globales. UAISCS-200-003.

Dougherty, B. (2008). Green Graphic Design. Nueva York: Allworth Press.

Edwards, W. (2011). Building the foundations of resilient communities. Public Management Magazine, septiembre 2011.

Fernández, V. (2014). Promoviendo un diseño urbano participativo: Experiencias desde la práctica y la docencia. Revista AUS, 15, 22-27. http://www.redalyc.org/articulo.oa? $i d=281732449005$

Foster, E. y Smith, C. (2015). Integrating resilience planning into university campus planning: Measuring risks and leveraging opportunities. Planning for Higher Education Journal, 44(1), 10-19.

Frascara, J. (2004). Diseño para la gente. Comunicación de masas y cambio social. Buenos Aires: Infinito.

Frascara, J. (2017). Design, and design education: How can they get together? Art, Design \& Communication in Higher Education, 16(1), 125-131, DOI: 10.1386/adch.16.1.125_1

Frye-Levine, L. (2012). Sustainability through design science: Reimagining option spaces beyond eco-efficiency. Sustainable Development, 20(3), 166-179. http://dx.doi.org/10.1002/sd.1533

Gallardo Milanés, O. (2014). Experiencias en la aplicación de la educación ambiental como herramienta para la adaptación al 
SIGraDi 2017, XXI Congreso de la Sociedad Ibero-americana de Gráfica Digital 22 - 24 Noviembre, 2017 - Concepción, Chile.

cambio climático en espacios comunitarios, en Holguín-Cuba. Sociedade \& Natureza, 26(2), 261-270. http://dx.doi.org/10.1590/1982-451320140205

Instituto Nacional de Ecología y Cambio Climático [INECC] (s.f.) El cambio climático en México. Secretaría del Medio Ambiente y Recursos Naturales (SEMARNAT). http://cambioclimatico.inecc.gob.mx/comprendercc/qpodemosha cerparamitigar/comoindividuos.html

Jacobson, S. (1997). Rapid Assessment for Conservation Education (RACE). The Journal of Environmental Education, 28(3), 10-19. http://dx.doi.org/10.1080/00958964.1997.9942824

Jowkar, B., Kojuri, J., Kohoulat, N., y Hayat, A. (2014). Academic Resilience in Education: The Role of Achievement Goal Orientations. Journal Of Advances In Medical Education And Professionalism, 2(1), 33-38.

Marcus, L., y Colding, J. (2014). Toward an integrated theory of spatial morphology and resilient urban systems. Ecology And Society, 19(4). http://dx.doi.org/10.5751/es-06939-190455

Moreno de las Casas, C. (2014). El diseño gráfico y su responsabilidad social. Revista Creatividad y Sociedad, 22, 1-15.
Organización de las Naciones Unidas [ONU] (2015). Convenio Marco sobre el Cambio Climático.http://unfccc.int/resource/docs/2015/cop21/spa/109s.pdf

Rendón, M. (2015). Universidad Verde y Sustentable. Un proyecto de Ateliori. Trabajo final del "Diplomado en Liderazgo Aplicado en Energías Renovables y Eficiencia Energética", impartido por la Escuela de Salud Pública de la Universidad de Harvard, InTrust Global Investment, Secretaría de Energía, con sede en la Universidad de Sonora. Hermosillo: Universidad de Sonora.

Sánchez, R. (2015). Vulnerability and Resiliency in the Built Environment. Boston: Harvard University.

Vale, L. (2013). The politics of resilient cities: whose resilience and whose city?. Building Research \& Information, 42(2), 191-201. http://dx.doi.org/10.1080/09613218.2014.850602

World Wildlife Fund [WWF] (2015). WWF presenta su campaña \#NiUnGradoMás para llamar a la acción contra el cambio climático. http://www.wwf.es/?35480/WWF-presenta-su-campaaNi-un-grado-ms--para-llamar-a-la-accin-contra-el-cambioclimatico 